\title{
Ageing and the Mediterranean diet: a review of the role of dietary fats
}

\author{
Maurizio Battino ${ }^{1, *}$ and Maria Soledad Ferreiro ${ }^{2}$ \\ ${ }^{1}$ Institute of Biochemistry, Faculty of Medicine, Università Politecnica delle Marche, Via Ranieri 65, \\ 1-60100 Ancona, Italy: ${ }^{2}$ Institute of Infectious Diseases and Public Heath, Faculty of Medicine, \\ Università Politecnica delle Marche, Italy
}

\begin{abstract}
Consumers are becoming increasingly aware of the relationship between food and health. Concerns have been raised about dietary fats and their relative nutritional advantages or disadvantages. In investigations of the associations between health and fat intake, special emphasis has been placed on the benefits of virgin olive oil for counteracting certain neurodegenerative diseases and ageing. With respect to ageing, accumulating evidence indicates that an improvement in quality of life can be reached by modulation of the extrinsic factors that influence many ageing processes. Of the modifiable factors, nutrition appears to be one of the strongest elements known to influence the rate of ageing as well as the incidence of age-associated diseases such as atherosclerosis and neurodegenerative pathologies.

This paper reviews the theory of ageing and the role of fatty acids in the mechanisms affecting its evolution. It also confirms that virgin olive oil, an essential component of the Mediterranean diet, provides large amounts of stable and not easily oxidisable fatty acids as well as remarkable quantities of powerful antioxidant molecules.
\end{abstract}

Keywords Oxidative stress Free radicals Antioxidants Mitochondria Virgin olive oil
Recent consumer interest in 'healthy eating' has raised awareness about the importance of limiting and selecting the consumption of fat and fatty foods. Current consumer questions to researchers include the following:

- What are the relative nutritional advantages and disadvantages of consuming fats?

- Are all fats bad for you?

- Since virgin olive oil appears as the unique fat source in recommended diets (e.g. the Mediterranean diet), could virgin olive oil be beneficial against ageing and for counteracting some neurodegenerative diseases?

- What is the biochemical rationale that allows this kind of speculation and can explain the numerous experimental and epidemiological data that seem to support such a hypothesis?

First of all, it should be considered what we currently and actually mean by ageing.

\section{Ageing: what it is, where and when it acts}

Ageing is a natural, multi-factorial process that occurs at cellular, organ and intact organism levels and affects most, but not all, living things. We used to believe that ageing was programmed in us by some kind of biological clock, but that view is no longer widely held. The most commonly accepted definition of ageing refers to a process that increases the vulnerability of an organism to challenges during its lifetime, thereby increasing the potential for death ${ }^{1}$. It is now thought that ageing is the result of accumulated damage to the cells and tissues in our bodies; in time, microscopic flaws impair normal functioning and may lead to disease. If we can understand cell ageing and find ways of reducing the accumulation of cell damage, or increase the effectiveness of our repair mechanisms, we might be able to delay the onset of disease and improve the quality of old age.

The individual's rate of ageing processes is influenced by both intrinsic and extrinsic factors: genetic make-up, lifestyle and environment. Accumulating evidence indicates that an improvement in the quality of life can be reached by modulation of the extrinsic factors that influence many ageing processes. The genetic make-up of individuals is pre-determined, and presently genetic manipulations cannot yet greatly influence the ageing rate of an individual. Nevertheless, lifestyle and environmental factors can interact with ageing processes and may alter the susceptibility of individuals to ageassociated diseases.

Of the environmental factors, nutrition appears to be one of the strongest factors known to influence the rate of ageing as well as the incidence of age-associated diseases (e.g. atherosclerosis and neurodegenerative diseases).

\section{Free radical (oxidative stress) theory of ageing}

Among the factors responsible for accumulated damage leading to ageing, we find several biological oxidants, 
arising from environmental sources (e.g. in the skin from exposure to ultraviolet light) or produced endogenously. Much of this cellular damage occurs as a by-product of normal living. For example, some of the oxygen we breathe gives rise to highly reactive molecules called free radicals, which can damage cell structures. Normal oxidation-reduction reactions are continuously producing trace amounts of free radicals. These highly unstable compounds contain one or more unpaired electrons in their outer shell and react with any susceptible compounds nearby, including DNA, lipids, proteins and carbohydrates $^{2,3}$. Although free radicals last only a few milliseconds, they can initiate a chain reaction resulting in the oxidation of thousands of particles over a great distance as the unpaired electron is transferred from one molecule to another in a game of 'hot potato ${ }^{2-4}$.

Our bodies have excellent natural defence mechanisms against these free radicals (i.e. the antioxidants) which minimise their unintended formation. Antioxidants may act at different cell sites and at different stages in the damage cascade induced by free radicals. They may prevent the formation of free radicals, neutralise them after they have been produced or, finally, repair the damage produced $^{2,5}$.

Oxidant attack and antioxidant defence are closely matched, but defences are inevitably less than perfect and damage caused by free radicals accumulates over time. It was proposed that this imbalance in favouzr of oxidants is a cause of ageing in itself, so that 'The ageing process may be simply the sum of random changes produced by [free radicall reactions, ${ }^{6}$.

\section{Biological membranes: essential and fragile structures}

All cellular structures can be considered targets for the action of free radicals. However, the structures that bear the highest amount of oxidative insults are actually all cell membranes (plasma membrane and any of the other organelle membranes). This happens mainly in the hydrophobic core of the membrane structure, which is constituted largely by fatty acids. The fatty acid domain is therefore the most susceptible to suffer dramatic damage induced by oxidative insults. The consequences provoked by changes in the membrane following an attack by free radicals affect both their molecular structure and their function.

Mitochondria, whose activities largely depend on the absolute integrity of the inner mitochondrial membrane, are particularly susceptible to any physiological, nutritional, pharmacological or environmental stimulus. Brain mitochondria are probably one of the best systems to study ageing.

In the last decade we have accumulated evidence indicating that ageing affects brain mitochondria to different extents depending on brain region and cellular district $^{7-11}$. This phenomenon is particularly evident at synaptic level and it is possible to study a specific population of aged mitochondria. The normal activities of these aged mitochondria are severely impaired: matrix activities (e.g. Krebs cycle) decrease, the electron transport chain works slowly and badly, and the trans-membrane systems that regulate the traffic of substrates across the inner membrane are also affected. The final results are a continuous leakage of electrons accompanied by the production of free radicals and partial uncoupling of the organelles that are unable to produce (enough) ATP, all this occurring in a continuous, vicious and lethal circle. Structurally, the membranes lose most of their antioxidants (like vitamin E and coenzyme Q) and finally also their typical shape is changed. In fact, they lose up to 75-80\% (Fig. 1a) of their fatty acids (mainly oleic acid), which are usually bound to membrane phospholipids, in this way greatly altering the classical aspect of the membrane (Fig. 1b).

It is not surprising, therefore, that treatments based on the administration of fatty acid mixtures are mediators and improve biochemical and cognitive functions ${ }^{12-15}$, and that a compound like phosphatidylserine, which contains two fatty acids per molecule, could have some efficacy in the treatment of certain neurodegenerative diseases.

a)

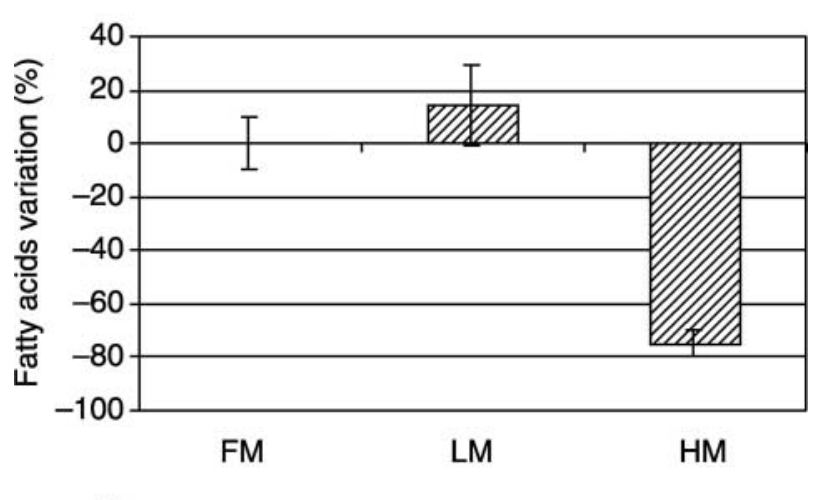

b)

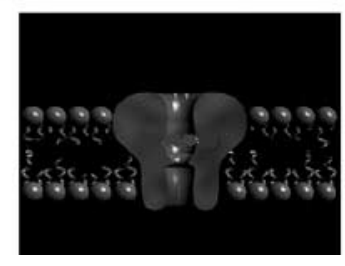

Fig. 1 (a) Percentage variation of fatty acids in brain mitochondria during ageing; FM are non-synaptic mitochondria taken as reference, LM are young synaptic mitochondria and HM are aged synaptic mitochondria. (b) Possible shape of the damaged membrane of aged synaptic mitochondria: the 'classical' ordered membrane structure is impaired as most phospholipid fatty acids have disappeared, been shortened or damaged; the mitochondrion is still alive but such new membrane organisation cannot support typical mitochondrial activities (mainly electron transfer and proton translocation) 
Moreover, not only do experimental data indicate that fatty acid supplementation is helpful in ameliorating cognitive status but also epidemiological evidence suggests different roles for specific fatty acids (like oleic acid) in protecting from cognitive decline ${ }^{16}$. Solfrizzi et al. studied the relationships between dietary macronutrient intakes and age-related changes in cognitive functions in an elderly population of southern Italy consuming a typical Mediterranean diet. They found that high intakes of monounsaturated fatty acids (MUFA) appeared to be protective against age-related cognitive decline and suggested this effect could be related to the role of these fatty acids in maintaining the structural integrity of neuronal membranes.

\section{Dietary olive oil: a tool for counteracting degenerative processes mediated by free radicals}

Dietary manipulation might be a wonderful tool for partially modifying the structure and consequently the features of biological membranes. The possibility of supporting cell membranes with those specific membrane components that are characterised by an elevated turnover allows one to ameliorate and/or counteract the typical modifications induced by the activity of free radicals. If the new fatty acids to be used in this process are provided in the form of virgin olive oil, the benefits are several and of different kinds. In fact, the peculiar origin of virgin olive oil (i.e. a 'fruit juice' and not a product of solvent extraction) results in a particularly enriched product characterised by two main components able to support either a passive or an active defence against attack by free radicals. On the one hand, virgin olive oil, with its very high content of MUFA (mainly oleic acid), greatly enriches all biological membranes so that it partially and gradually substitutes the other fatty acids, mainly polyunsaturated fatty acids (PUFA). This process produces membranes that are less susceptible to oxidative injury because of the increased concentration of oleic acid, which - while maintaining an optimal fluidity for membrane functionality - at the same time slows down the propagation of lipid peroxidation phenomena that usually require the fatty acids to possess two or more double bonds ${ }^{17}$. On the other hand, virgin olive oil also contains a large amount of antioxidant molecules (like $\alpha$-tocopherol, several different phenolic compounds and even coenzyme Q). These molecules are well-known free-radical scavengers that neutralise the toxic species and sometimes even prevent the early steps of their formation, in this way protecting biological structures from oxidative insult ${ }^{18-20}$. The actual efficacy of dietary interventions based on virgin olive oil, as a unique source of fat for modifying the fatty acid pattern of biological membranes making them less prone to suffer oxidative modifications, has been widely demonstrated $^{21-24}$. Also, in the past decade we have largely clarified several molecular mechanisms involved in these phenomena in animals, demonstrating that dietary administration of virgin olive oil leads to beneficial changes in not only plasma but also in the microsomes and mitochondria of different tissues, even under extremely stressful conditions (xenobiotic administration, acute and chronic physical exercise, pharmacological treatment, etc. $)^{25-39}$.

As far as the effect of such a diet on humans is concerned, we have studied the possibility of a similar dietary intervention on hypercholesterolaemic (type IIb) patients. The rationale for this investigation lies in the wellestablished, direct and indirect relationships existing between atherosclerosis and plasma lipid status.

In fact, decades of research have clearly demonstrated that diet has a strong influence on plasma lipids and lipoprotein levels, with dietary fats being the factors most directly implicated ${ }^{40}$. Investigations concerning the relationships between plasma cholesterol level and the quantity/quality of dietary fats revealed the cholesterolraising action of saturated fatty acids (SFA). This class of lipids was beneficially substituted by MUFA or PUFA for decreasing plasma total cholesterol and low-density lipoprotein (LDL)-cholesterol concentrations ${ }^{21,41}$. Despite the ability of diets enriched with PUFA to reduce plasma cholesterol level, the degree of unsaturation in these fatty acids influences the susceptibility of LDL to undergo oxidative modifications, making these particles more prone to peroxidation and in principle more atherogenic. When MUFA substitute SFA, they can reduce concentrations of total cholesterol and LDL-cholesterol without affecting high-density lipoprotein-cholesterol, which in turn is efficiently lowered by PUFA. In addition, MUFA lead to favourable changes in the lipid profile of lipoproteins, generating LDL particles more resistant to oxidative modifications ${ }^{42}$.

According to the above findings, interest has recently been focused on the benefits of the Mediterranean diet on hyperlipidaemia and other established cardiovascular risk factors ${ }^{43}$. The Mediterranean diet is characterised by a low content of SFA and a concomitant abundant intake of MUFA in the form of virgin olive oil. Furthermore, high MUFA intake may combine the advantages of both lowering cholesterol levels and decreasing LDL susceptibility to oxidation. This aspect could be crucial because lipoprotein oxidation is now widely accepted as a contributing factor in the pathogenesis of atherosclerosis ${ }^{44}$.

Lipid oxidation leads to a chain reaction and to various kinds of aldehyde as final products. The interaction of these compounds with apolipoprotein B is probably critical in generating a form that is taken up by macrophages, via the scavenger receptor, from the bloodstream into the subendothelial space. The scavenger receptor leads to an unlimited uptake by macrophages of LDL that has undergone oxidative modifications, which 
finally become lipid-loaded foam cells. These latter contribute to the formation of fatty streaks and subsequently to plaque formation ${ }^{45}$.

We carried out a specific investigation studying the effects of feeding virgin olive oil versus a PUFA-enriched diet on LDL composition and features in hypercholesterolaemic (type IIb) patients ${ }^{46,47}$. The diets clearly affected LDL composition, with MUFA enhancement of $10 \%$ and PUFA decrease of $11 \%$ brought about by the diet containing virgin olive oil. The most interesting data were obtained when LDL particles were considered for their capability to resist different peroxidation insults. The challenge of oleate-enriched LDL with $\mathrm{Cu}^{2+}$ yielded a lower lag phase $(P<0.05)$ in the production of conjugated dienes (Fig. 2) and the same LDL gave lower lipid hydroperoxide contents after exposure to AAPH (a thermally dependent free-radical initiator).

It was demonstrated, therefore, that LDL composition could be greatly modified by dietary intervention, obtaining in this way lipoproteins that are less prone to undergoing oxidative alterations (i.e. a way to produce less atherogenic LDL). Again, virgin olive oil can support considerable amounts of both poorly oxidisable fatty acids and, at the same time, several antioxidant species.

Therefore, taking into account that (1) the integrity and functionality of all cell membranes depend largely on the membrane's capacity for counteracting free radical insults by maintaining a particular shape and composition, and (2) daily nutrient intake greatly affects the composition of cell membranes and lipoproteins and it is possible to change this composition within a few weeks of dietary treatment, we can conclude that specific dietary intervention might be a new, interesting and promising challenge in the treatment (and mainly prevention) of several age-related diseases, as a useful adjuvant to the corresponding pharmacological therapy. Moreover, it should be underlined that in this kind of strategy an outstanding role is played by virgin olive oil

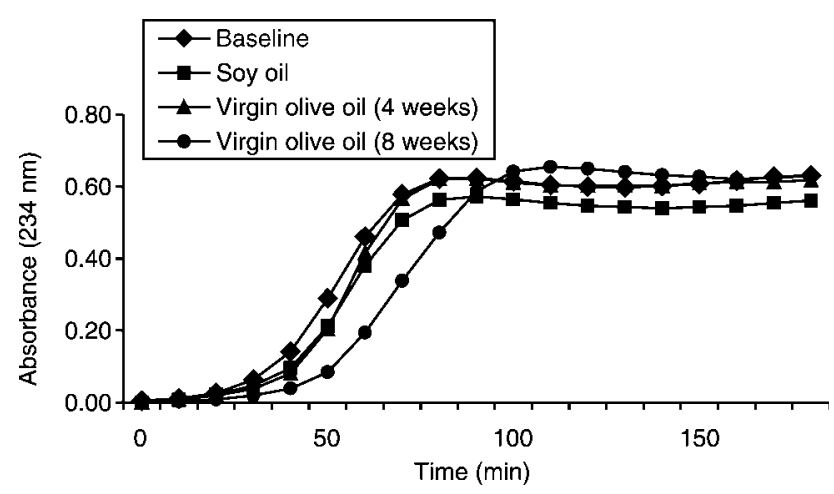

Fig. 2 Formation of conjugated dienes during $\mathrm{Cu}^{2+}$ oxidation of low-density lipoprotein (LDL) from hypercholesterolaemic (type IIb) patients. After 8 weeks on a diet enriched with virgin olive oil, LDL particles from these patients were significantly less oxidisable than at recruitment or when following a diet enriched with soy oil (see text for more details) per se, which - being an essential component of the Mediterranean diet - can provide large amounts of stable and not easily oxidisable fatty acids as well as remarkable quantities of powerful antioxidant molecules.

\section{Acknowledgements}

The studies from the authors' laboratory were partially supported by Ancona University and F. Ili Carli SpA (Imperia, Italy). The large and fruitful collaborations with Professor RF Villa and Dr A Gorini (University of Pavia, Italy) and the group lead by Professor J Mataix at Granada University (Spain) are gratefully acknowledged. Special thanks go to Ms Monica Glebocki for preparation of the manuscript.

\section{References}

1 Massoro EJ. Concepts and hypothesis of basic aging process In: Yu BP, ed. Free Radicals in Aging. Boca Raton, FL: CRC Press, 1993; $1-9$.

2 Battino M, Bullon P, Wilson M, Newman HN. Oxidative injury and inflammatory periodontal diseases: the challenge of anti-oxidants to free radicals and reactive oxygen species. Critical Reviews in Oral Biology and Medicine 1999; 10: 458-76.

3 Battino M, Ferreiro MS, Gallardo I, Newman HN, Bullon P. The antioxidant capacity of saliva. Journal of Clinical Periodontology 2002; 29: 189-94.

4 Gaziano JM. Dietary antioxidants and cardiovascular disease. Vitamins and Hormones 2000; 58: 299-320.

5 Niki E. $\alpha$-Tocopherol. In: Cadenas E, Packer L, eds Handbook of Antioxidants. New York: Marcel Dekker, 1996; 3-25.

6 Harman D. Role of free radicals in aging and disease. Annals of the New York Academy of Sciences 1992; 673: 126-41.

7 Battino M, Bertoli E, Formiggini G, Sassi S, Gorini A, Villa RF, et al. Structural and functional aspects of the respiratory chain of synaptic and non synaptic mitochondria derived from selected brain regions. Journal of Bioenergetics and Biomembranes 1991; 23: 345-63.

8 Battino M, Gorini A, Villa RF, Genova ML, Bovina C, Sassi S, et al. Coenzyme Q content in synaptic and nonsynaptic mitochondria from different brain regions in the ageing rat. Mechanisms of Ageing and Development 1995; 78: $173-87$.

9 Battino M, Littarru GP, Gorini A, Villa RF. Coenzyme Q, peroxidation and cytochrome oxidase features after Parkinson's-like disease by MPTP toxicity in intra-synaptic and non-synaptic mitochondria from Macaca fascicularis cerebral cortex and hippocampus: action of dihydroergocriptine. Neurochemical Research 1996; 21: 1505-14.

10 Battino M, Quiles JL, Huertas JR, Mataix JF, Villa RF, Gorini A. Cerebral cortex synaptic heavy mitochondria may represent the oldest synaptic mitochondrial population: biochemical heterogeneity and effects of L-acetylcarnitine. Journal of Bioenergetics and Biomembranes 2000; 32 $163-73$.

11 Battino M, Ferreiro MS, Littarru GP, Quiles JL, RamirezTortosa MC, Huertas JR, et al. Structural damage induced by peroxidation may account for functional impairment of heavy synaptic mitochondria. Free Radical Research 2002; 36: 479-84.

12 Yehuda S, Rabinovitz S, Carasso RL, Mostofsky DI. Fatty acid 
mixture counters stress changes in cortisol, cholesterol, and impair learning. International Journal of Neuroscience 2000; 101: 73-87.

13 Yehuda S, Rabinovitz S, Mostofsky DI. Essential fatty acids are mediators of brain biochemistry and cognitive functions. Journal of Neuroscience Research 1999; 56: 565-70.

14 Yehuda S, Rabinovitz S, Mostofsky DI. Modulation of learning and neuronal membrane composition in the rat by essential fatty acid preparation: time-course analysis. Neurochemical Research 1998; 23: 627-34.

15 Yehuda S, Rabinovitz S, Mostofsky DI, Huberman M, Sredni B. Essential fatty acid preparation improves biochemical and cognitive functions in experimental allergic encephalomyelitis rat. European Journal of Pharmacology 1997; 328: 23-9.

16 Solfrizzi V, Panza F, Torres F, Mastroianni F, Del Parigi A, Venezia A. High monounsaturated fatty acids intake protects against age-related cognitive decline. Neurology 1999; 52: 1563-9.

17 Landvik SV, Diplock AT, Packer L. Efficacy of vitamin E in human health and disease. In: Cadenas E, Packer L, eds. Handbook of Antioxidants. New York: Marcel Dekker, 1996; 63-87.

18 Grignaffini P, Roma P, Galli C, Catapano AL. Protection of low density lipoprotein from oxidation by 3,4-dihydroxyphenylethanol. Lancet 1994; 343: 1296-7.

19 Visioli F, Bellom G, Montedoro GF, Galli C. Low density lipoprotein oxidation is inhibited in vitro by olive oil constituents. Atherosclerosis 1995; 117: 25-32.

20 Petroni A, Blasevich M, Salami M, Papini N, Montedoro GF, Galli C. Inhibition of platelet aggregation and eicosanoid production by phenolic components of olive oil. Thrombosis Research 1995; 78: 151-60.

21 Mattson FH, Grundy SM. Comparison of effects of dietary saturated, monounsaturated and polyunsaturated fatty acids on plasma lipids and lipoproteins. Journal of Lipid Research 1985; 26: 194-202.

22 Giron MD, Mataix J, Suarez MD. Changes in lipid composition and desaturase activity of duodenal mucosa induced by dietary fat. Biochimica et Biophysica Acta 1990; 1045: 69-73.

23 Periago JL, Suarez MD, Pita ML. Effect of dietary olive oil, corn oil, and medium-chain triglycerides on the lipid composition of rat red blood cell membranes. Journal of Nutrition 1990; 120: 986-94.

24 Seiquer I, Manas M, Martinez-Victoria E, Huertas JR, Ballesta MC, Mataix FJ. Effects of adaptation to diets enriched with saturated, monounsaturated and polyunsaturated fats on lipid and serum fatty acid levels in miniature swine (Sus scrofa). Comparative Biochemistry and Physiology 1994; 108A: $377-86$.

25 Huertas JR, Battino M, Lenaz G, Mataix FJ. Changes in mitochondrial and microsomal rat liver coenzyme Q9 and Q10 content induced by dietary fat and endogenous lipid peroxidation. FEBS Letters 1991; 287: 89-92.

26 Huertas JR, Battino M, Mataix FJ, Lenaz G. Cytochrome oxidase induction after oxidative stress induced by adriamycin in liver of rats fed with dietary olive oil. Biochemical and Biophysical Research Communications 1991; 181: 375-82.

27 Huertas JR, Battino M, Barzanti V, Maranesi M, ParentiCastelli G, Littarru GP, et al. Mitochondrial and microsomal cholesterol mobilization after oxidative stress induced by adriamycin in rats fed with dietary olive and corn oil. Life Sciences 1992; 50: 2111-8.

28 Quiles JL, Huertas JR, Manas M, Battino M, Cassinello M, Littarru GP, et al. Peroxidative extent and coenzyme Q levels in the rat: influence of physical training and dietary fats. Molecular Aspects of Medicine 1994; 15(Suppl.): S89-95.

29 Barzanti V, Battino M, Baracca A, Cavazzoni M, Cocchi M,
Noble R, et al. The effect of dietary lipid changes on the fatty acid composition and function of liver, heart and brain mitochondria in the rat at different ages. British Journal of Nutrition 1994; 71: 193-202.

30 Huertas JR, Mataix FJ, Manas M, Bargossi AM, Battino M. Dietary polyunsaturated fatty acids and peroxidative risks in sport practice. Alternatives. Journal of Sports Medicine and Physical Fitness 1994; 34: 101-8.

31 Mataix J, Manas M, Quiles J, Battino M, Cassinello M, LopezFrias M, et al. Coenzyme Q content depends upon oxidative stress and dietary fat unsaturation. Molecular Aspects of Medicine 1997; 18(Suppl.): S129-35.

32 Mataix J, Quiles JL, Huertas JR, Battino M, Manas M. Tissue specific interactions of exercise, dietary fatty acids, and vitamin $\mathrm{E}$ in lipid peroxidation. Free Radical Biology $\varepsilon$ Medicine 1998; 24: 511-21.

33 Quiles JL, Huertas JR, Manas M, Battino M, Ochoa JJ, Mataix J. Plasma antioxidants are strongly affected by iron-induced lipid peroxidation in rats subjected to physical exercise and different dietary fats. Biofactors 1998; 8: 119-27.

34 Quiles JL, Huertas JR, Manas M, Battino M, Mataix J. Physical exercise affects the lipid profile of mitochondrial membranes in rats fed with virgin olive oil or sunflower oil. British Journal of Nutrition 1999; 81: 21-4.

35 Quiles JL, Ramirez-Tortosa MC, Huertas JR, Ibanez S, Gomez JA, Battino M, et al. Olive oil supplemented with vitamin E affects mitochondrial coenzyme Q levels in liver of rats after an oxidative stress induced by adriamycin. Biofactors 1999; 9: $331-6$.

36 Quiles JL, Huertas JR, Manas M, Ochoa JJ, Battino M, Mataix $\mathrm{J}$. Oxidative stress induced by exercise and dietary fat modulates the coenzyme $\mathrm{Q}$ and vitamin A balance between plasma and mitochondria. International Journal for Vitamin and Nutrition Research 1999; 69: 243-9.

37 Quiles JL, Huertas JR, Manas M, Ochoa JJ, Battino M, Mataix J. Dietary fat type and regular exercise affect mitochondrial composition and function depending on specific tissue in the rat. Journal of Bioenergetics and Biomembranes 2001; 33: $127-34$.

38 Battino M, Quiles JL, Huertas JR, Ramirez-Tortosa MC, Cassinello M, Manas M, et al. Feeding fried oil changes antioxidant and fatty acid pattern of rat and affects rat liver mitochondrial respiratory chain components. Journal of Bioenergetics and Biomembranes 2002; 34: 127-34.

39 Quiles JL, Battino M, Huertas JR, Ramirez-Tortosa MC, Cassinello M, Mañas M, et al. The intake of fried virgin olive or sunflower oils differentially induces oxidative stress in rat liver microsomes. British Journal of Nutrition 2002; 88: 57-65.

40 Grundy SM, Denke MA. Dietary influences on serum lipids and lipoproteins. Journal of Lipid Research 1990; 31: 1149-72.

41 Mensik RP, Katan MB. Effect of a diet enriched with monounsaturated or polyunsaturated fatty acids on levels of low density and high density lipoprotein cholesterol in healthy women and men. New England Journal of Medicine 1989; 321: 436-41.

42 Parthasarathy S, Khoo JC, Miller E, Barnett J, Witztum JL, Steinberg D. Low density lipoprotein rich in oleic acid is protected against oxidative modification: implications for dietary prevention of atherosclerosis. Proceedings of the National Academy of Sciences of the USA 1990; 87: 3894-8.

43 International Consensus Statement. Olive oil and the Mediterranean diet: implications for health in Europe. Meeting Convened by the European Commission at the Italian National Research Council, Rome, 11 April 1997.

44 Witztum JL, Steinberg D. Role of oxidised low density lipoprotein in atherogenesis. Journal of Clinical Investigation 1991; 88: 1785-92.

45 Steinberg D, Parthasarathy S, Carew TE, Khoo JC, Witztum 
JL. Beyond cholesterol. Modification of low density lipoprotein that increase its atherogenity. New England Journal of Medicine 1989; 320: 915-24.

46 Svegliati Baroni S, Amelio M, Fiorito A, Gaddi A, Littarru G, Battino M. Monounsaturated diet lowers LDL oxidisability in type IIb and type IV dyslipidemia without affecting coenzyme Q10 and vitamin E contents. Biofactors 1999; 9 325-30.

47 Baroni SS, Amelio M, Sangiorgi Z, Gaddi A, Battino M. Solid monounsaturated diet lowers LDL unsaturation trait and oxidisability in hypercholesterolemic (type IIb) patients. Free Radical Research 1999; 30: 275-85. 